\title{
Psychometric Evaluation of the Persian Translation of the Thalassemia-Specific Quality of Life Questionnaire
}

\author{
Mohsen Adib-Hajbaghery, PhD \\ Kashan University of Medical Sciences, Kashan, Iran \\ Saeed Poormansouri, MSc \\ Mehrnaz Ahmadi \\ Ahvaz Jundishapur University of Medical Sciences, Ahvaz, Iran
}

\begin{abstract}
Background and Purpose: There is no Persian scale for assessing the quality of life of patients with thalassemia major. Thus, this study was conducted to translate and validate the 36-item thalassemia-specific Transfusion-Dependent Quality of Life (TranQoL) questionnaire. Methods: The questionnaire was first translated into Persian and then was backward-translated into English. Afterward, panels of experts commented on the Persian translation, and then its face validity was confirmed and content validity index (CVI) and content validity ratio (CVR) were calculated. The factorial structure and the reliability of the translated questionnaire were assessed using exploratory factor analysis (EFA) and internal consistency method, respectively. Results: The CVI and CVR of the questionnaire were .95 and .84 , respectively. Five items were deleted during EFA, and 9 factors were extracted from the 31 remaining items. The reliability of the questionnaire was confirmed through a Cronbach's alpha coefficient of .9. Conclusion: The Persian translation of the TranQoL questionnaire is highly reliable and valid, and thus, it can be used as a specific measure for assessing the quality of life among patients with thalassemia.
\end{abstract}

Keywords: quality of life; thalassemia major; instrument development; validity; reliability

$\mathrm{T}$ halassemia refers to a group of hereditary blood diseases caused by defects in the gene responsible for the production of globin chains. It is one of the most common genetic diseases across the world (Boonchooduang, Louthrenoo, Choeyprasert, \& Charoenkwan, 2015; Higgs, Engel, \& Stamatoyannopoulos, 2012). It is prevalent in indigenous African communities, the Indian subcontinent, the Mediterranean region, as well as the Middle East and Southeast Asia (Khodaei, Farbod, \& Saeidi, 2013; Razzazan, Ravanipour, Gharibi, Motamed, \& Zarei, 2014). There are about 300 million patients with thalassemia around the world. Thalassemia is also a common health problem in our country, Iran, particularly in the northern and the southern regions of the country (Khodaei et al., 2013). Currently, with more than 20,000 patients with thalassemia and 3 million carriers of the gene, Iran is in the global thalassemia belt (Centers for Disease Control and Prevention \& Ministry of Health and Medical Education, 2006; Thavorncharoensap et al., 2010). 
Thalassemia is a chronic disease that causes many physical and psychological problems for the patients. It leads to a wide range of health, social, and financial problems that affect the life of both the patients and their families (Adib-Hajbaghery, Ahmadi, \& Poormansouri, 2015; Khodaei et al., 2013). Previous studies have shown that patients with thalassemia major have a poorer quality of life (QOL) compared to the general population (Boonchooduang et al., 2015; Gupta \& Jindal, 2016).

As an important component of health, QOL comprises a set of emotional and cognitive reactions to physical, psychological, and social conditions. It is always regarded as the final outcome in clinical trials and health care services (Zeraatkar, Rahimian Boogar, Talepasand, \& Amin, 2016). It is also a key indicator of health among patients with chronic conditions (Lyrakos, Vini, Aslani, \& Drosou-Servou, 2012). Many attempts have been made over the past three decades to objectively define and measure QOL (Issazadegan, Sepehrianazar, \& Matlabi, 2016) and many general and specific instruments have been developed for its assessment.

Most studies have used the 36-item Short-Form Survey (SF-36) to assess QOL in patients with thalassemia major (Haghpanah et al., 2013; Khani et al., 2012; Porter et al., 2012). SF-36 is a general QOL assessment scale that is used nonspecifically to assess the QOL of different patient populations (Safizadeh, Farahmandinia, Nejad, Pourdamghan, \& Araste, 2012; Trachtenberg et al., 2014; Yengil et al., 2014; Zarea, Baraz Pordanjani, Pedram, \& Pakbaz, 2012). However, it does not cover the specific problems of patients with thalassemia. Blood transfusion and iron chelation therapy are among the factors that can affect QOL among patients with thalassemia (Adib-Hajbaghery et al., 2015; Boonchooduang et al., 2015; Haghpanah et al., 2013; Porter et al., 2012; Trachtenberg et al., 2014); however, general QOL assessment instruments do not address these factors. Besides, patients with thalassemia major suffer from problems such as physical disability and functional limitations. Moreover, they face different familial, educational, and occupational challenges which in turn can lead to numerous psychological problems and poor QOL (Adib-Hajbaghery et al., 2015; Khoury et al., 2012; Shafiee, Nazari, Jorjani, Bahraminia, \& Sadeghi-Koupaei, 2014; Zeighami Mohammadi, Tajvidi, \& Ghazizadeh, 2014).

\section{BACKGROUND AND CONCEPTUAL FRAMEWORK}

Given the development of new treatment processes, nurses and all health care providers are ethically and professionally obliged to evaluate patient outcomes such as QOL. QOL assessment among patients with thalassemia major requires new and specific instruments to help accurately identify subtle but very important factors that affect QOL (Klaassen et al., 2014).

Most studies emphasized the poorer QOL among patients with thalassemia compared to others (Ansari, Baghersalimi, Azarkeivan, Nojomi, \& Hassanzadeh Rad, 2014; Caocci et al., 2012; Haghpanah et al., 2013; Kaheni et al., 2013; Khaledi, Moridi, \& Valiee, 2013; Khani et al., 2012; Safizadeh et al., 2012; Zarea et al., 2012). However, these studies neither sufficiently addressed factors behind thalassemia-related QOL nor discussed its particular dimensions. Such gaps might have been caused by the lack of a thalassemiaspecific instrument for comprehensively assessing QOL and its various dimensions. Therefore, the development and the use of new specific instruments seem essential to better identify these factors and develop better social and health policies for the prevention, early diagnosis, and treatment of different thalassemia-related health problems. 
The Transfusion-Dependent Quality of Life questionnaire (TranQoL) was developed by a group of Canadian experts to specifically assess QOL among patients with thalassemia (Klaassen et al., 2014). One strength of this questionnaire-compared to general QOL questionnaires - is that it construes the specific problems faced by patients with thalassemia as the factors affecting their QOL. These problems are the effect of thalassemia on school and career functioning, distress about the need for regular blood transfusion and the likelihood of catching infectious diseases in the process, the painful tests, concerns about childbearing, the discomfort and limitations caused by regular iron chelation therapy, as well as fear of early death because of the disease and sexual activities. In a study in Canada, Klaassen et al. (2013) assessed and confirmed the validity and reliability of this instrument (Klaassen et al., 2013). The TranQoL has four versions for different age groups including children and adolescents, adults, parents, and caregivers. Given the lack of Persian thalassemia-specific QOL assessment instruments, this study was conducted to translate and validate the adult version of TranQoL.

\section{METHODS}

\section{Design}

This multistage cross-sectional methodological study was carried out in 2015.

\section{The Transfusion-Dependent Quality of Life Questionnaire}

TranQol was primarily designed to assess the QOL of patients who need frequent blood transfusions. The 36 items of the questionnaire measure the respondent's QOL over the past week. These items cover five domains, including physical health, emotional health, family functioning, sexual activities, and school and career functioning. Each item is scored from 0 to 100 based on a 5-point scale (Never, Rarely, Occasionally, Mostly, and Always). The first objective of this study was to translate the questionnaire and produce a Persian translation to be linguistically and semantically compatible with the English version. Thus, we first obtained permission from the developer of TranQoL questionnaire via e-mail and then translated it into Persian language in the steps proposed by the International Quality of life Assessment project (Aaronson et al., 1992).

\section{Translation of the Original Instrument}

First, the original English TranQoL was translated into Persian language by two independent English-Persian translators. Then, a meeting was held between the translators, and the researchers and necessary modifications were made to develop an initial Persian version of the questionnaire. After that, two Persian-English translators translated the initial Persian translation back into English. Then, meetings were held between the researchers and the translators to compare this English version to the original English TranQoL. The conceptual inconsistencies were discussed in these meetings, and necessary modifications were made to the Persian translation.

\section{Face Validity Assessment}

In a preliminary assessment of the face validity, the questionnaire was distributed among 10 nurses who had a minimal thalassemia-related work experience of 2 years, 6 nursing 
instructors who had some thalassemia-related work experience, as well as 10 patients with thalassemia major. They were asked to comment on the intelligibility and clarity of the items. Ten other experts were also asked to comment on the grammar, wording, item placement, and scoring of the Persian TranQoL. Their views were assessed in a meeting held among the authors, and ambiguities in the questionnaire were clarified. Finally, instructions on how to complete the questionnaire were added to the beginning of the questionnaire.

\section{Content Validity Assessment}

The content validity index (CVI) of the questionnaire was assessed according to the method proposed by Waltz and Bausell (Waltz, Strickland, \& Lenz, 2010), and its content validity ratio (CVR) was assessed according to Lawshe's method (Lawshe, 1975). Therefore, the Persian translation of the TranQoL questionnaire was distributed among 20 faculty members of Ahvaz Jundishapur University of Medical Sciences, Ahvaz, Iran. They were asked to comment on the necessity, relevance, simplicity, and clarity of each item. They were eight individuals with a doctor of philosophy $(\mathrm{PhD})$ degree in nursing, four $\mathrm{PhD}$ nursing students, and eight instructors with a master's degree in nursing. Moreover, six of them had the experience of working in thalassemia wards or a history of teaching students about thalassemia.

The necessity of including each of the items was determined according to CVR. Accordingly, the experts were asked to rate each item on a 3-point scale (necessary, useful but not necessary, and not necessary). The responses were then entered into the following equation: $\mathrm{CVR}=(n \mathrm{E}-N / 2) /(N / 2)$, where $n$ is the number of experts who deemed the item necessary and $N$ the total number of the experts. The total CVR was measured by calculating the mean CVR of all items. Based on Lawshe's table and given the number of the experts $(N=20)$, items with a CVR of more than .42 were deemed acceptable (Baheiraei et al., 2013).

To determine the CVI of the questionnaire, the experts were asked to determine the relevance, simplicity, and clarity of each item on a 4-point Likert scale. For instance, the relevance of each item was assessed using the following four points: totally relevant (4), somewhat relevant (3), needs serious revision (2), and irrelevant (1). The CVI of each item was calculated by dividing the number of experts who scored that item either 3 or 4 by the total number of the experts. The total CVI for each item (I-CVI) was obtained according to the mean CVI of all three aspects of CVI, whereas the total scale CVI (S-CVI) was obtained through calculating the mean CVI of all items (Polit \& Beck, 2006).

\section{Construct Validity Assessment}

The exploratory factor analysis was used to assess the construct validity of the instrument. Only those items with a factor loading greater than .3 were used in the exploratory factor analysis. The factors were extracted according to the correlation matrix of the variables. The study sample consisted of 154 patients with thalassemia major admitted to Shafa Hospital, Ahvaz, Iran. Because the study population was limited, through the census method, all eligible patients with thalassemia major who are aged 18 years or older were included. Initially, necessary permissions were obtained from the Research Deputies of Kashan and Jundishapur Universities of Medical Sciences, Kashan and Ahvaz, Iran. Then, the second researcher referred to the Hematology Clinic of Shafa Hospital, Ahvaz, Iran, and distributed the questionnaire among all eligible patients. The patients were asked to answer the items according to the instructions provided at the beginning of the questionnaire as well as the verbal information provided by the researcher. The questionnaire was a self-administered instrument. Those participants who were literate enough personally completed TranQoL, 
whereas those who were illiterate $(n=1)$ or barely literate $(n=23)$ responded to the items with the help of the researcher and through the interview technique. The second author (SP) was present in the research setting in all stages of data collection.

The Kaiser-Meyer-Olkin (KMO) test was used in the exploratory factor analysis to assess the adequacy of the sample size. KMO test value was .82, suggesting a good factor analysis. Moreover, the suitability of the correlation matrix was assessed using Bartlett's test of sphericity. The test value was 2,242.72 $(p<.001)$ and therefore, the factor analysis model was appropriate. The factor analysis model explained $66 \%$ of the variance of thalassemia-related QOL.

\section{Reliability Assessment}

To assess the reliability of the questionnaire, the internal consistency method was used and Cronbach's alpha coefficient was calculated to assess the internal consistency of the entire questionnaire as well as for each of its subscales. Pearson's correlation coefficient was also used to determine the correlation between the subscale scores and the overall score. Data were analyzed in SPSS (Version 16.0) at a significance level of .05.

\section{Ethical Considerations}

Ethical approval for the study was received from the research ethics committee of Kashan University of Medical Sciences, Kashan, Iran (the code was IR.KAUMS.REC.1394.135). Also, the study was granted by the university research deputy (the grant number was 94134). In addition, required permissions were received from relevant authorities in Jundishapur University of Medical Sciences and the Hematology Clinic of Shafa Hospital, Ahvaz, Iran. All participants in the study signed a written informed consent form and were assured of the confidentiality of their personal information and absence of any constraint to participate in the study.

\section{RESULTS}

One hundred fifty-four patients with thalassemia major (84 women and 70 men) with a mean age of $25.74 \pm 5.72$ years participated in this study. The majority of the participants $(85.7 \%)$ were single. Table 1 presents the demographic characteristics of the participants. The mean and standard deviation of the QOL scores are presented in Table 2.

\section{Translation and Face Validity}

All items of the questionnaire were found to have a good face validity. After making the necessary adjustments as per the experts' comments, the items of the Persian translation of TranQoL questionnaire were deemed relevant, simple, and clear enough with respect to grammar, wording, item placement, and scoring and thus, no more changes were made to the items.

\section{Content Validity}

In total, 20 experts and faculty members commented on the content validity of the questionnaire. A CVI of .79 or higher was deemed acceptable for the different aspects of the items. The I-CVI of the items ranged from .8 to 1 , and the S-CVI was .95. The lowest CVI obtained (.8) pertained to the simplicity aspect of the Items 5 and 8 , and the lowest CVI (.85) pertained to the relevance and the clarity aspects of the Items 5, 6, and 8. Because the minimum acceptable CVI was .79, none of the items were excluded. Yet, the Items 5 and 6 
TABLE 1. Demographic Features of the Study Participants

\begin{tabular}{lcc}
\hline Demographic Profile & Frequency & $\%$ \\
\hline Gender & & \\
$\quad$ Female & 84 & 54.5 \\
Male & 70 & 45.5 \\
Marital status & 132 & \\
$\quad$ Single & 21 & 85.7 \\
Married & 1 & 13.6 \\
Divorced & & 0.6 \\
Ethnicity & 57 & \\
Arab & 94 & 37 \\
Fars & 3 & 61 \\
Others & & 1.9 \\
Education level & 24 & 46.6 \\
$\quad<$ High school diploma & 72 & 37.6 \\
High school diploma & 58 & \\
College & & 53.9 \\
Employment & 83 & 28.5 \\
Unemployed & 44 & 14.8 \\
Employed & 27 & \\
Student & & \\
\hline
\end{tabular}

were modified to include clear examples of heavy and moderate activities. Moreover, the phrase "physical activity" was added to the Item 8 to improve respondents' understanding of its meaning. Table 3 presents CVI results.

A CVR of .42 and higher for the entire test was deemed acceptable. The CVR of the TranQoL items ranged from .6 to 1 . The lowest CVR (.6) pertained to the Items 8 and 19 , and hence, there was no need to eliminate any of the items. All items had a favorable content validity and remained in the instrument. Necessary modifications were made

TABLE 2. The Mean and Standard Deviation of Quality of Life Score and Its Dimensions in Patients With Thalassemia Major

\begin{tabular}{lc}
\hline Quality of Life Scales & $M \pm S D$ \\
\hline Physical health & $61.34 \pm 14.61$ \\
Emotional health & $57.22 \pm 18.35$ \\
Sexual activity & $52.53 \pm 36.16$ \\
Family functioning & $59.09 \pm 19.59$ \\
School and career functioning & $57.30 \pm 31.99$ \\
Overall quality of life score & $58.87 \pm 15.82$ \\
\hline
\end{tabular}


TABLE 3. Relevance, Simplicity, Clarity, Item Content Validity Index (I-CVI), and Content Validity Ratio (CVR) Values of the 36-Item Farsi Draft of Transfusion-Dependent Quality of Life Questionnaire

\begin{tabular}{|c|c|c|c|c|c|}
\hline Items & Relevance & Simplicity & Clarity & $\mathrm{I}-\mathrm{CVI}$ & CVR \\
\hline 1. I had trouble sleeping. ${ }^{\mathrm{a}}$ & 1.00 & 1.00 & .95 & .98 & 1.00 \\
\hline 2. I was free of pain or discomfort. ${ }^{\mathrm{a}}$ & 1.00 & 1.00 & 1.00 & 1.00 & 0.80 \\
\hline 3. Pain prevented me from doing what I need to do. & 1.00 & 1.00 & 1.00 & 1.00 & 1.00 \\
\hline 4. I had enough energy for daily activities. & 1.00 & .95 & .90 & .95 & 1.00 \\
\hline 5. I was limited in my ability to do the kind of moderate work I take part in. ${ }^{\mathrm{a}}$ & .85 & .80 & .85 & .83 & .80 \\
\hline 6. I was limited in my ability to do the kind of vigorous work I take part in. & .85 & .90 & .85 & .86 & .80 \\
\hline $\begin{array}{l}\text { 7. I felt too tired to do the things I enjoy doing (i.e., hobbies, crafts, sports, } \\
\text { musical instruments). }\end{array}$ & .95 & .95 & .95 & .95 & .70 \\
\hline 8. I found it hard to keep up with my peers. & .85 & .80 & .85 & .83 & 60 \\
\hline 9. My health allows me to participate in as many social events as I would like. & .95 & .95 & 1.00 & .96 & .90 \\
\hline 10. My treatments prevent me from doing the things I want to do. & 1.00 & 1.00 & .95 & .98 & .90 \\
\hline 11. I felt sad. & 1.00 & 1.00 & 1.00 & 1.00 & 1.00 \\
\hline 12. I worried about getting an infection from the blood I receive. & .95 & 1.00 & .95 & .96 & .90 \\
\hline 13. I worried about becoming sicker. & 1.00 & .95 & .95 & .96 & 1.00 \\
\hline 14. I felt angry. ${ }^{\mathrm{b}}$ & 1.00 & .95 & .90 & .95 & 90 \\
\hline 15. I felt anxious. ${ }^{b}$ & 1.00 & 1.00 & .95 & .98 & .90 \\
\hline 16. I was hopeful about my future. & .95 & 1.00 & 1.00 & .98 & .90 \\
\hline 17. I felt comfortable telling a significant other about my condition. ${ }^{\mathrm{a}}$ & .90 & 1.00 & .95 & .95 & .80 \\
\hline 18. I worried thalassemia may lead to an earlier death. & .95 & 1.00 & .95 & .96 & .80 \\
\hline 19. I worried about painful tests. & .90 & 0.90 & .90 & .90 & .60 \\
\hline 20. I worried about being able to have children. & 1.00 & 1.00 & 1.00 & 1.00 & 1.00 \\
\hline
\end{tabular}


21. I was bothered by my transfusion.

\begin{tabular}{rrrrr}
1.00 & 1.00 & 1.00 & 1.00 & 1.00 \\
1.00 & .95 & .95 & .96 & 1.00 \\
.95 & .90 & .90 & .91 & .70 \\
1.00 & 1.00 & 1.00 & 1.00 & 1.00 \\
.95 & 1.00 & 1.00 & .98 & .70 \\
.95 & .95 & .95 & .95 & .80 \\
.80 & 1.00 & 1.00 & .93 & .70 \\
.95 & .95 & .95 & .95 & 1.00 \\
.85 & 1.00 & 1.00 & .95 & .70 \\
1.00 & 1.00 & 1.00 & 1.00 & .80 \\
.95 & .95 & .95 & .95 & .70 \\
& & & & \\
.95 & .95 & .95 & .95 & .70 \\
.90 & .90 & .90 & .9 & .70 \\
.95 & .95 & .95 & .95 & .90 \\
.95 & .95 & .95 & .95 & .90 \\
.90 & .90 & .90 & .90 & .90 \\
& & & $.95^{\mathrm{c}}$ & .84 \\
\hline
\end{tabular}

34. I have had trouble keeping up with my work, or I do not work.

35. I am bothered because I missed work, ${ }^{\mathrm{b}}$ or I do not work.

36. Thalassemia limited my career opportunities.

Total

I was bothered by my iron removal treatment.

25. I am satisfied with my sex life.

26. I received enough support from my family.

28. Thalassemia negatively affected my family.

29. I worried about paying for my medication/equipment.

0. My treatments posed a financial strain for me/my family

1. I have had trouble keeping up with my schoolwork, or I did not attend school.

32. Thalassemia and its treatments affect my academic performance, or I did not attend school.

${ }^{a}$ These items were deleted in factor analysis.

'These items were rephrased according to the experts' comments.

${ }^{\mathrm{c} S} \mathrm{~S}$ ale validity content index (S-CVI). 
according to the experts' comments regarding the phrasing and the wording of each item. For example, Items 14, 15, and 24 were written in full to clarify some ambiguities, whereas Items 26 and 27 were further clarified to address the issue of family and peer support. The responses to Items 33 and 35 were modified to read "I did not leave school" and "I did not quit my work" to remove ambiguities. Also, given the logical sequence of the effect of illness on school functioning, the order of Items 31 and 32 were switched.

\section{Construct Validity}

To determine the construct validity of the instrument and the number of factors, exploratory factor analysis was used. Consequently, those items with the highest correlation with each other were grouped into nine factors. Figure 1 presents the scree plot resulting of the exploratory factor analysis. The nine factors were physical functioning (four items), role limitations because of physical health (three items), anxiety and worry (six items), treatment-related fatigue (two items), emotional problems (three items), social support/ hope (three items), family (three items), and school and career functioning (six items). Factor nine included just one item to assess patients' sexual health. At this stage, five items $(1,2,5,17$, and 23 in the original questionnaire) were eliminated because of conceptual incompatibility with the extracted factors. Hence, the 31 items remaining in factor analysis were loaded on one of the nine factors. The factors of physical functioning and role limitations because of physical health were considered as the subscale of physical health, whereas the factors of anxiety and worry, treatment-related fatigue, and emotional problems were considered as the subscale of emotional health to match the categories in the original questionnaire. Thus, it can be declared that the factors extracted in this study were largely similar to those in the original questionnaire. Table 4 presents the factor loading of each item based on the rotated factor matrix of the TranQoL items.

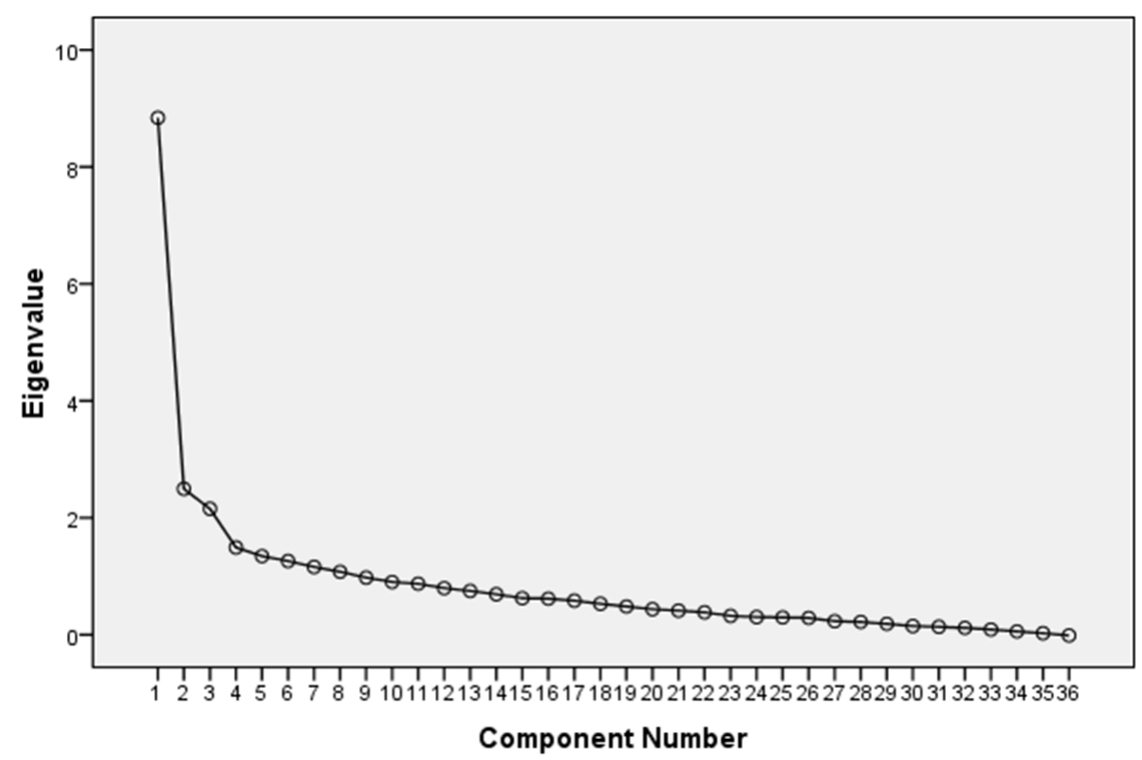

Figure 1. The scree plot results of the exploratory factor analysis. 
TABLE 4. Factors Loading for the Nine Extracted Subscales After Varimax Rotation

\begin{tabular}{|c|c|c|c|c|c|c|c|c|c|c|}
\hline Items' Number ${ }^{\mathrm{a}}$ & Subscales and Items & 1 & 2 & 3 & 4 & 5 & 6 & 7 & 8 & 9 \\
\hline 4 & I had enough energy for daily activities. & -.698 & & & & & & & & \\
\hline 8 & $\begin{array}{l}\text { I found it hard to keep up physical activity with my } \\
\text { peers. }\end{array}$ & .658 & & & & & & & & \\
\hline 9 & $\begin{array}{l}\text { My health allows me to participate in as many social } \\
\text { events as I would like. }\end{array}$ & -.551 & & & & & & & & \\
\hline 3 & Pain prevented me from doing what I need to do. & .495 & & & & & & & & \\
\hline 7 & $\begin{array}{l}\text { I felt too tired to do the things I enjoy doing (i.e., } \\
\text { hobbies, crafts, sports, musical instruments). }\end{array}$ & & .510 & & & & & & & \\
\hline 6 & $\begin{array}{l}\text { I was limited in my ability to do the kind of vigorous } \\
\text { work I take part in (such as running, lifting heavy } \\
\text { objects, participating in strenuous sports). }\end{array}$ & & .372 & & & & & & & \\
\hline 10 & $\begin{array}{l}\text { My treatments prevent me from doing the things } \\
\text { I want to do. }\end{array}$ & & .303 & & & & & & & \\
\hline 12 & $\begin{array}{l}\text { I worried about getting an infection from the blood } \\
\text { I receive }\end{array}$ & & & .757 & & & & & & \\
\hline 20 & I worried about being able to have children. & & & .694 & & & & & & \\
\hline 13 & I worried about becoming sicker. & & & .653 & & & & & & \\
\hline 18 & I worried thalassemia may lead to an earlier death. & & & .445 & & & & & & \\
\hline 19 & I worried about painful tests. & & & .437 & & & & & & \\
\hline $15^{\mathrm{b}}$ & $\begin{array}{l}\text { I felt more anxious than other people in similar } \\
\text { situations. }\end{array}$ & & & .382 & & & & & & \\
\hline 22 & I was bothered by my iron removal treatment. & & & & .775 & & & & & \\
\hline 21 & I was bothered by my transfusion. & & & & .750 & & & & & \\
\hline $14^{\mathrm{b}}$ & I felt angry more often than other people. & & & & & .715 & & & & \\
\hline
\end{tabular}


TABLE 4. Factors Loading for the Nine Extracted Subscales After Varimax Rotation (Continued)

\begin{tabular}{|c|c|c|c|c|c|c|c|c|c|c|}
\hline Items' Number ${ }^{\mathrm{a}}$ & Subscales and Items & 1 & 2 & 3 & 4 & 5 & 6 & 7 & 8 & 9 \\
\hline $24^{\mathrm{b}}$ & $\begin{array}{l}\text { I felt different from other people because of my disease } \\
\text { complications and limitations. }\end{array}$ & & & & & .530 & & & & \\
\hline 11 & I felt sad. & & & & & .473 & & & & \\
\hline $26^{\mathrm{b}}$ & My family supports me and my health problems. & & & & & & 696 & & & \\
\hline $27^{\mathrm{b}}$ & My friends support me and my health problems. & & & & & & .685 & & & \\
\hline 16 & I was hopeful about my future. & & & & & & .636 & & & \\
\hline 30 & $\begin{array}{l}\text { My treatments posed a financial strain for me/my } \\
\text { family (i.e., transportation to/from hospital, } \\
\text { equipment, time off work). }\end{array}$ & & & & & & & .761 & & \\
\hline 28 & Thalassemia negatively affected my family. & & & & & & & 642 & & \\
\hline 29 & I worried about paying for my medication/equipment. & & & & & & & 634 & & \\
\hline 32 & $\begin{array}{l}\text { Thalassemia and its treatments affect my academic } \\
\text { performance, or I did not attend school. }\end{array}$ & & & & & & & & .764 & \\
\hline 34 & $\begin{array}{l}\text { I have had trouble keeping up with my work, or I do } \\
\text { not work. }\end{array}$ & & & & & & & & .753 & \\
\hline 31 & $\begin{array}{l}\text { I have had trouble keeping up with my schoolwork, or } \\
\text { I did not attend school. }\end{array}$ & & & & & & & & .740 & \\
\hline 36 & Thalassemia limited my career opportunities. & & & & & & & & .712 & \\
\hline $33^{\mathrm{b}}$ & $\begin{array}{l}\text { I am bothered because I missed school. or I did not } \\
\text { leave school. }\end{array}$ & & & & & & & & .682 & \\
\hline $35^{\mathrm{b}}$ & $\begin{array}{l}\text { I am bothered because I missed work. or I did not quit } \\
\text { my work. }\end{array}$ & & & & & & & & 606 & \\
\hline 25 & I am satisfied with my sex life. & & & & & & & & & .665 \\
\hline
\end{tabular}

aFive items (Items 1, 2, 5, 17, and 23 in the original Transfusion-dependent Quality of Life [TranQoL] questionnaire) were eliminated.

'btems 14, 15, 24, 26, 27, 33 and 35 were rephrased. 
TABLE 5. Pearson Correlation Coefficient Between the Scores of the Subscales and Total Score of Quality of Life

\begin{tabular}{lc}
\hline Subscales of Quality of Life & Correlation Coefficient, $p$ Value \\
\hline Physical health & \\
$\quad \begin{array}{l}\text { Physical functioning } \\
\quad \text { Role limitations due to physical health }\end{array}$ & $r=.73, p>.001$ \\
Emotional health & \\
$\quad$ Anxiety and worry & $r=.79, p>.001$ \\
$\quad$ Fatigue related to treatments & $r=.66, p>.001$ \\
$\quad$ Emotional problems & $r=.75, p>.001$ \\
Social support/hope & $r=.45, p>.001$ \\
Family functioning & $r=.67, p>.001$ \\
School and career functioning & $r=.78, p>.001$ \\
Sexual health & $r=.40, p>.001$
\end{tabular}

\section{Reliability}

The Cronbach's alpha coefficients of the Persian TranQoL were as follows: whole TranQoL $=.9$; physical health $=.7$; emotional health $=.85$; school and career functioning $=.85$; family $=.72$; social support/hope $=.64$. It was impossible to examine the reliability of the sexual health factor because it contained only one item. The Pearson's correlation coefficients showed a favorable correlation between the subscales and the total score of TranQoL (Table 5). As shown in Table 5, the total score of the Persian TranQoL had a significant positive correlation with all subscales.

\section{DISCUSSION}

In recent years, many studies have been conducted to examine QOL among patients with thalassemia, particularly regarding the effect of nursing interventions and services. Conducting proper studies on this subject requires valid and specific instruments or scales because patients with thalassemia major have unique problems and concerns that are less addressed in general QOL instruments. For instance, although one of the greatest concerns of patients with thalassemia and their families is about infection because of regular blood transfusion and use of blood products, the general SF-36 survey includes no item in this area (Klaassen et al., 2014). However, the thalassemia-specific TranQoL questionnaire assesses the effect of illness on the family and includes items on different aspects of the disease. Factors such as blood transfusion and the subsequent iron overload (Adib-Hajbaghery et al., 2015; Boonchooduang et al., 2015; Haghpanah et al., 2013; Porter et al., 2012; Trachtenberg et al., 2014), organ dysfunction, chronic pain, and the loss of physical fitness create problems that affect QOL in patients with thalassemia (Pakbaz et al., 2005). The TranQoL questionnaire adequately addresses all these problems. The unique aspects of the disease and the specific problems faced by patients necessitate the use of a specific instrument for measuring thalassemia-related QOL. Because no specific 
instruments exist in Persian for measuring QOL in patients with thalassemia major, translating the specific instrument available on the subject and validating its content appeared necessary.

This study showed that the Persian translation of TranQoL questionnaire has a good face and content validity. This instrument was translated in this study by expert translators with a mastery over the process of translation and with special attention to the crosscultural compatibility of the concept.

Face validity assessment showed that the items of the Persian TranQoL were clear, simple, and intelligible enough. The results of content validity assessment (CVR $=.84$ and $\mathrm{S}-\mathrm{CVI}=.95)$ revealed the questionnaire had acceptable content validity.

Exploratory factor analysis revealed that the Persian TranQoL questionnaire consisted of nine factors. Some items of the original TranQoL were eliminated from the Persian translation because of their conceptual incompatibility with the extracted factors. These items were "I had trouble sleeping," "I was free of pain or discomfort," "I was limited in my ability to do the kind of moderate work I take part in," "I felt comfortable telling a significant other about my condition," and "I felt guilty about missing my treatments."

The original questionnaire contains 36 items in five dimensions, including physical health, emotional health, sexual activity, family functioning, and school and career functioning (Klaassen et al., 2013; Klaassen et al., 2014). In this study, the "physical functioning" and the "role limitations due to physical health" dimensions were considered as the subscales of the "physical health" dimension, whereas the "anxiety and worry," "fatiguerelated treatment," and "emotional problems" were considered as the subscales of the "emotional health." The "family" and "social support" dimensions can also be considered the subscales of the "family functioning." Item 16 of the instrument, that is, "I was hopeful about my future," had a strong correlation with the two items which assessed social support; therefore, this dimension was named "social support/hope." The other dimensions matched the dimensions in the original questionnaire.

The Cronbach's alpha coefficients of the TranQoL and its dimensions were .9 and $.7-.85$, respectively. These coefficients showed a favorable reliability of the questionnaire. However, the coefficient of the "social support/hope" dimension was .64. Similarly, Klaassen et al. found that the alpha coefficient of the questionnaire and its dimensions were .96 and .74-.88, which suggest an overall good reliability (Klaassen et al., 2014). The Pearson's correlation coefficients also showed a significant positive correlation between the total score of TranQoL and the scores of its different dimensions, indicating the good internal consistency of the test.

A limitation of this study was that it did not examine the criterion validity of the questionnaire; yet, the developers of TranQoL reported a significantly high correlation between the TranQoL and SF-36 questionnaire (Klaassen et al., 2014). Then, further studies are recommended to evaluate the criterion validity of the Persian TranQoL.

In this study, a sample of 154 patients with thalassemia was recruited for factor analysis. Sample size is a matter of paramount importance to sound exploratory factor analysis. According to Hair et al. (as cited in Williams, Onsman, \& Brown, 2010) sample size should be 100 or greater. Other researchers focused on the number of cases per item. For instance, Cattell (as cited in Gaskin \& Happell, 2014) noted that 3-20 cases per item are needed for factor analysis. However, in many cases, especially in medical research projects, it is very difficult to collect a large sample of patients suffering from a certain disease (Jung \& Lee, 2011). Although the KMO test confirmed the adequacy of sample size in this study, replicating the same study on a larger sample of patients with thalassemia can 
produce convincing results regarding the appropriateness of the Persian TranQoL. In addition, designing context-based instruments for assessing the thalassemia-related QOL is recommended. Such a context-based instrument might be more congruent with the culture and the specific problems of Iranian patients with thalassemia major.

\section{IMPLICATIONS IN NURSING}

Nurses are responsible for assessing and improving the QOL and the health status of their patients, especially those with chronic disorders such as thalassemia. Thus, they need to have sufficient knowledge about various aspects of these patients' lives of. The Persian TranQoL not only can be used by the researchers for QOL assessment among patients with thalassemia major but also can be used by nurses to diagnose and manage the unique problems of these patients. Nursing instructors can also use the Persian TranQoL questionnaire to teach their students the specific problems affecting the QOL of patients with thalassemia.

\section{CONCLUSION}

In this study we translated the 36-item TranQoL questionnaire and evaluated its psychometric properties. Based on the results of exploratory factor analysis, the number of TranQoL items was reduced to 31. The Persian TranQoL has six dimensions, namely physical health, emotional health, family functioning, social support/hope, school and career functioning, and sexual health. The Persian TranQoL has acceptable psychometric properties. Thus, it is recommended to be used as a good thalassemia-specific QOL assessment instrument. TranQoL questionnaire addresses the unique problems experienced by patients with thalassemia, and hence, it can help health care providers, such as nurses, develop care plans for managing these patients' problems. Yet, further studies are needed to produce convincing results regarding the appropriateness of the Persian TranQoL. Moreover, designing a context-based thalassemia-specific instrument is suggested.

\section{REFERENCES}

Aaronson, N. K., Acquadro, C., Alonso, J., Apolone, G., Bucquet, D., Bullinger, M., .. . Ware, E. J., Jr. (1992). International Quality of Life Assessment (IQOLA) project. Quality of Life Research, 1(5), 349-351.

Adib-Hajbaghery, M., Ahmadi, M., \& Poormansouri, S. (2015). Health related quality of life, depression, anxiety and stress in patients with beta-thalassemia major. Iranian Journal of Pediatric Hematology and Oncology, 5(4), 193-205.

Ansari, Sh., Baghersalimi, A., Azarkeivan, A., Nojomi, M., \& Hassanzadeh Rad, A. (2014). Quality of life in patients with thalassemia major. Iranian Journal of Pediatric Hematology and Oncology, 4(2), 57-63.

Baheiraei, A., Khoori, E., Ahmadi, F., Foroushani, A. R., Ghofranipour, F., \& Weiler, R. M. (2013). Psychometric properties of the Adolescent Health Concern Inventory: The Persian version. Iranian Journal of Psychiatry, 8(1), 28-36.

Boonchooduang, N., Louthrenoo, O., Choeyprasert, W., \& Charoenkwan, P. (2015). Health-related quality of life in adolescents with thalassemia. Pediatric Hematology and Oncology, 32(5), 341-348. http://dx.doi.org/10.3109/08880018.2015.1033795 
Caocci, G., Efficace, F., Ciotti, F., Roncarolo, M. G., Vacca, A., Piras, E., . . La Nasa, G. (2012). Health related quality of life in Middle Eastern children with beta-thalassemia. BMC Blood Disorders, 12, 6. http://dx.doi.org/10.1186/1471-2326-12-6

Centers for Disease Control and Prevention \& Ministry of Health and Medical Education. (2006, October 11, 2017). Information and Statistics of Noncontagious Diseases in Iran. Retrieved from http://darman.fums.ac.ir/upload/15/07/06/4-1_94_gozaresh\%20bimarihaye\%20gheir\%20 vagiri.pdf

Gaskin, C. J., \& Happell, B. (2014). On exploratory factor analysis: A review of recent evidence, an assessment of current practice, and recommendations for future use. International Journal of Nursing Studies, 51(3), 511-521.

Gupta, M., \& Jindal, R. (2016). Quality of life in patients with thalassemia major. International Journal of Science and Research, 5(5), 41-42.

Haghpanah, S., Nasirabadi, S., Ghaffarpasand, F., Karami, R., Mahmoodi, M., Parand, S., \& Karimi, M. (2013). Quality of life among Iranian patients with beta-thalassemia major using the SF-36 questionnaire. Sao Paulo Medical Journal, 131(3), 166-172.

Higgs, D. R., Engel, J. D., \& Stamatoyannopoulos, G. (2012). Thalassaemia. Lancet, 379(9813), 373-383. http://dx.doi.org/10.1016/s0140-6736(11)60283-3

Issazadegan, A., Sepehrianazar, F., \& Matlabi, M. (2016). Comparing the purpose of life and quality of life, in cancerous patients and healthy individuals. Journal of Urmia Nursing and Midwifery Faculty, 13(12), 1097-1103.

Jung, S., \& Lee, S. (2011). Exploratory factor analysis for small samples. Behavior Research Methods, 43(3), 701-709.

Kaheni, S., Yaghobian, M., Sharefzadah, G. H., Vahidi, A., Ghorbani, H., \& Abderahemi, A. (2013). Quality of life in children with $\beta$-thalassemia major at Center for Special Diseases. Iranian Journal of Pediatric Hematology and Oncology, 3(3), 108-113.

Khaledi, S., Moridi, G., \& Valiee, S. (2013). Comparison the quality of life of healthy and thalassemic children. Iranian Journal of Nursing Research, 8(2), 87-94.

Khani, H., Majdi, M. R., Marzabadi, E. A., Montazeri, A., Ghorbani, A., \& Ramezani, M. (2012). Quality of life of Iranian beta-thalassaemia major patients living on the southern coast of the Caspian Sea. Eastern Mediterranean Health Journal, 18(5), 539-545.

Khodaei, G. H., Farbod, N., \& Saeidi, M. (2013). Frequency of thalassemia in Iran and Khorasan Razavi. International Journal of Pediatrics, 1(1), 45-50.

Khoury, B., Musallam, K. M., Abi-Habib, R., Bazzi, L., Ward, Z. A., Succar, J., . . . Taher, A. T. (2012). Prevalence of depression and anxiety in adult patients with $\beta$-thalassemia major and intermedia. International Journal of Psychiatry in Medicine, 44(4), 291-303.

Klaassen, R. J., Alibhai, S., Allen, M. K., Moreau, K., Pulcini, M. M., Forgie, M. M., . . Young, N. L. (2013). Introducing the Tran Qol: A new disease-specific quality of life measure for children and adults with thalassemia major. Journal of Blood Disorders and Transfusion, 4(4), 1-5.

Klaassen, R. J., Barrowman, N., Merelles-Pulcini, M., Vichinsky, E. P., Sweeters, N., Kirby-Allen, M., . . Y Young, N. L. (2014). Validation and reliability of a disease-specific quality of life measure (the TranQol) in adults and children with thalassaemia major. British Journal of Haematology, 164(3), 431-437. http://dx.doi.org/10.1111/bjh.12631

Lawshe, C. H. (1975). A quantitative approach to content validity. Personnel Psychology, 28, 563-575.

Lyrakos, G. N., Vini, D., Aslani, H., \& Drosou-Servou, M. (2012). Psychometric properties of the Specific Thalassemia Quality of Life Instrument for adults. Patient Preference and Adherence, 6, 477-497. http://dx.doi.org/10.2147/ppa.s30763

Pakbaz, Z., Treadwell, M., Yamashita, R., Quirolo, K., Foote, D., Quill, L., . . Vichinsky, E. P. (2005). Quality of life in patients with thalassemia intermedia compared to thalassemia major. Annals of the New York Academy of Sciences, 1054, 457-461. http://dx.doi.org/10.1196/ annals. 1345.059

Polit, D. F., \& Beck, C. T. (2006). The content validity index: Are you sure you know what's being reported? Critique and recommendations. Research in Nursing \& Health, 29(5), 489-497. http://dx.doi.org/10.1002/nur.20147

Porter, J., Bowden, D. K., Economou, M., Troncy, J., Ganser, A., Habr, D., . . C Cappellini, M. D. (2012). Health-related quality of life, treatment satisfaction, adherence and persistence in $\beta$-thalassemia and myelodysplastic syndrome patients with iron overload receiving 
deferasirox: Results from the EPIC clinical trial. Anemia, 2012, 297641. http://dx.doi.org/ $10.1155 / 2012 / 297641$

Razzazan, N., Ravanipour, M., Gharibi, T., Motamed, N., \& Zarei, A. (2014). Effect of selfmanagement empowering model on the quality of life in adolescents and youths with major thalassemia. Journal of Nursing Education, 3(2), 48-52.

Safizadeh, H., Farahmandinia, Z., Nejad, S. S., Pourdamghan, N., \& Araste, M. (2012). Quality of life in patients with thalassemia major and intermedia in kerman-iran (I.R.). Mediterranean Journal of Hematology and Infectious Diseases, 4(1), e2012058. http://dx.doi.org/10.4084/ mjhid.2012.058

Shafiee, A., Nazari, S., Jorjani, S., Bahraminia, E., \& Sadeghi-Koupaei, M. (2014). Prevalence of depression in patients with $\beta$-thalassemia as assessed by the Beck's Depression Inventory. Hemoglobin, 38(4), 289-291. http://dx.doi.org/10.3109/03630269.2014.929008

Thavorncharoensap, M., Torcharus, K., Nuchprayoon, I., Riewpaiboon, A., Indaratna, K., \& Ubol, B. O. (2010). Factors affecting health-related quality of life in Thai children with thalassemia. BMC Blood Disorders, 10, 1. http://dx.doi.org/10.1186/1471-2326-10-1

Trachtenberg, F. L., Gerstenberger, E., Xu, Y., Mednick, L., Sobota, A., Ware, H., . . . Yamashita, R. (2014). Relationship among chelator adherence, change in chelators, and quality of life in thalassemia. Quality of Life Research, 23(8), 2277-2288. http://dx.doi.org/10.1007/s11136-014-0671-2

Waltz, C. F., Strickland, O., \& Lenz, E. R. (2010). Measurement in nursing and health research. New York, NY: Springer Publishing.

Williams, B., Onsman, A., \& Brown, T. (2010). Exploratory factor analysis: A five-step guide for novices. Australasian Journal of Paramedicine, 8(3), 990399.

Yengil, E., Acipayam, C., Kokacya, M. H., Kurhan, F., Oktay, G., \& Ozer, C. (2014). Anxiety, depression and quality of life in patients with beta thalassemia major and their caregivers. International Journal of Clinical and Experimental Medicine, 7(8), 2165-2172.

Zarea, K., Baraz Pordanjani, Sh., Pedram, M., \& Pakbaz, Z. (2012). Quality of life in children with thalassemia who referred to thalassemia center of shafa hospital. Jundishapur Journal of Chronic Disease Care, 1(1), 45-53.

Zeighami Mohammadi, Sh., Tajvidi, M., \& Ghazizadeh, Sh. (2014). The relationship between spiritual well-being with quality of life and mental health of young adults with beta-thalassemia major. Scientific Journal of Iran Blood Transfus Organ, 11(2), 147-154.

Zeraatkar, M., Rahimian Boogar, I., Talepasand, S., \& Amin, A. (2016). The effectiveness of healthy lifestyle promotion intervention on quality of life in patients with congestive heart failure via cognitive-behavioral procedure. Journal of Shahid Sadoughi University of Medical Sciences, 23(11), 1094-1107.

Acknowledgments. We are thankful of the research deputy of Kashan University of Medical Sciences for their supports. We also are thankful of faculty members of Ahvaz Jundishapur University of Medical Sciences and authorities and staff in Thalassemia clinic of Ahwaz Shafa Hospital for their kind cooperation in this study. We also acknowledge the sincere cooperation of all patients with thalassemia major who participated in this study.

Correspondence regarding this article should be directed to Mohsen Adib-Hajbaghery, PhD, Kashan University of Medical Sciences, Trauma Nursing Research Center, Faculty of Nursing and Midwifery, Ravand Road, Kashan, Iran. E-mail: adib1344@yahoo.com 